\title{
CA215, A New Pan Cancer Biomarker, and its Clinical Applications
}

\author{
Gregory Lee Le,2* $^{1,2}$ \\ ${ }^{1}$ UBC Center for Reproductive Health, Vancouver, Canada \\ ${ }^{2}$ Shantou University Medical College, Shantou, China
}

Received: 20 June, 2015; Accepted: 01 September, 2015; Published: 20 September, 2015

*Corresponding author: Gregory Lee, UBC Center for Reproductive Health, University of British Columbia, 9117 Shaughnessy Street, Vancouver, Canada V6P 6R9, Tel.: 778-322-4651; Fax: 604-325-4677, E-mail address: leecyg@gmail.com

\begin{abstract}
CA215 is a tumor-associated antigen which originated from the generation of a monoclonal antibody, RP215, against cultured ovarian cancer cell extract in 1987. To identify the molecular identity of CA215, MALDI-TOF MS studies were performed for affinity-isolated CA215 from shed medium of cultured OC-3-VGH ovarian cancer cells. RP215 was shown to react with a carbohydrate-associated epitope located mainly on the immunoglobulin heavy chains (designated in general as CA215) expressed by most cancer cells. By using RP215 as a probe, both sandwich and competitive immunoassays were formulated to determine the serum CA215 levels among patients diagnosed with known cancers of the lung, liver, colon, esophagus, ovary, breast, pancreas, cervix, and lymphocytes. When each of the other known cancer biomarkers, such as AFP, CEA, CA 125, CA 19-9, CA 15-3, and Cyfra 21-1 were combined with CA215, it was generally demonstrated that much higher cancer detection rates were observed, as compared to the use of a single biomarker alone. Therefore, CA215, which consists mainly of cancerous immunoglobulins, was clearly demonstrated as a pan cancer biomarker for potential immunodiagnostic applications of human cancer.
\end{abstract}

Keywords: CA215; RP215; Cancerous immunoglobulins; Sandwich enzyme immunoassay; Competitive enzyme immunoassay; Pan cancer biomarkers; Cancer immunodiagnostics; Carbohydrateassociated epitope

\section{Introduction}

In 1987, a monoclonal antibody designated as RP215 was generated against the OC-3-VGH ovarian cancer cell extract [15]. This monoclonal antibody has been extensively characterized with respect to the molecular nature of its cognate antigen, CA215, from different cancer cell lines and tissue specificity by immunohistochemical studies [2]. Through initial studies, it was generally concluded that the antigen recognized by RP215 is highly associated with cancer cells or cancerous tissues, but it is not found on normal human tissues, except in hyperplastic epithelial cells or tissues, such as those of the skin, esophagus, and cervix, as well as in several immune-privileged sites, including the neuron, eye, and testis [1-4,6-10].
The antigen recognized by RP215, CA215, can be detected in the shed media from a number of cultured cancer cells $[2,5]$. Therefore, Matrix-Assisted Laser Desorption/Ionization TimeOf-Flight Mass Spectrometry (MALDI-TOF MS) analysis was performed to determine the molecular identity of affinityisolated CA215 from the shed media of OC-3-VGH ovarian and C-33A cervical cancer cell lines [2,5]. Following tryptic digestion of CA215, the peptide fragments were subjected to analysis by MALDI-TOF MS. It was observed that as many as $50 \%$ of the peptide fragments derived from the shed media of cultured cancer cells were found to have a high degree of homology to antigen receptors, including immunoglobulins $(42 \%)$ and $\mathrm{T}$ cell receptors (6\%) expressed by cancer cells [5]. Many other different glycoproteins generally categorized as immunoglobulin superfamily proteins (IgSF) were also detected in such analyses [11].

In view of the observation that the immunoactivity of the RP215-specific epitope in CA215 is destroyed by periodate treatments under mild conditions at neutral $\mathrm{pH}$, it was assumed that the unique RP215 epitope is carbohydrate-associated [3]. Following extensive studies through glycopeptide mapping and glycosyl linkage analysis, it was suggested that the RP215specific epitope may be associated with a simple 0-linked glycan together with amino acid residues in the variable region of cancerous immunoglobulin heavy chains [12]. But this RP215specific epitope is not found in immunoglobulins expressed by normal B cells [3].

Since CA215 is cancer-associated, elevated levels of CA215 in the human circulation may indicate active growth/proliferation or metastasis of cancer cells in the human body [1,2,5,11,13-20]. Immunoassays using RP215 as a unique substitute for antibodies against cancerous immunoglobulins have been established for the quantitative determination of serum levels of CA215 [1]. They have successfully been used to monitor CA215 levels in the human sera of patients confirmed or diagnosed with cancers of various tissue origins [21].

Therefore, in this review, results of CA215 clinical evaluations are highlighted to demonstrate the potential applications of 
CA215 as a pan cancer biomarker. Parallel comparisons with other known cancer biomarkers are also presented to document the clinical utility of CA215 in the immunodiagnostics of many different types of cancer in humans [21]. Meanwhile several review papers from the author's lab have also been published and should facilitate our understanding about the potential functional roles of cancerous immunoglobulins or CA215, as well as the potential applications of RP215 in cancer immunotherapy [22-26].

\section{Methods}

\section{Sandwich enzyme immunoassays for CA215}

Basically two different RP215-based immunoassay procedures can be performed to quantitatively determine CA215 levels from cancer patients during routine clinical diagnostic applications [1]. They are sandwich and competitive immunoassays, respectively. CA215 is a mixture of different glycoproteins, including immunoglobulins, expressed by cancer cells, each of which is attached or linked with the RP215-specific carbohydrate-associated epitope(s) [11]. Therefore, RP215 can serve as a unique probe to tag cancerous immunoglobulins and can be used to formulate immunoassays for the quantitation of CA215. If a given CA215 contains two or more repeated RP215specific epitopes, a sandwich enzyme immunoassay can be designed based on the use of RP215 monoclonal antibody alone as the capturing and detecting antibody with enzyme labeling, such as with Horseradish peroxidase (HRP). This sandwich enzyme immunoassay has been employed for a large scale evaluations of serum CA215 levels of more than 500 patients confirmed with different cancers [21]. CA215 antigen standards were required for clinical evaluations of CA215 levels among cancer patients and were prepared from partially purified CA215 obtained from the shed medium of cultured OC-3-VGH ovarian cancer cells. Similar to many other tumor biomarkers, the CA215 cancerassociated antigen was calibrated and defined in arbitrary units per millimeter $(\mathrm{AU} / \mathrm{mL})$. The typical CA215 sandwich enzyme immunoassay is a two hour single-step procedure at $37^{\circ} \mathrm{C}$ with a minimum detection sensitivity of $0.02 \mathrm{AU} / \mathrm{mL}$. Positive CA215 levels are defined as those above the mean reading of normal negative serum plus two standard deviations [21].

\section{Competitive enzyme immunoassay for CA215}

An alternative competitive enzyme immunoassay method was also formulated for the quantitative determination of CA215 as the pan cancer biomarker. In a typical competitive enzyme immunoassay, microwells are coated with partially purified CA215. RP215 conjugated with HRP served as the signal detecting antibody. This immunoassay is initiated by adding serum specimens containing CA215 to compete with well-coated CA215 for the binding of RP215-HRP conjugate in the incubation mixture. Therefore, after this one hour assay procedure, the signal intensity can be observed and is inversely proportional to the CA215 found in a given patient serum specimen. A typical standard curve in this competitive assay is presented in Figure 1 for comparative purposes.

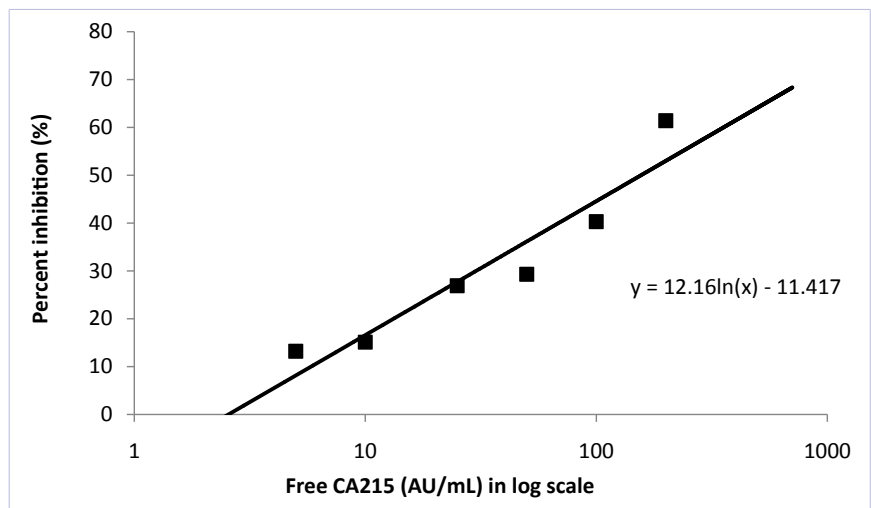

Figure 1: Standard curve of typical competitive CA215 immunoassay in log scale of free CA215 (free AU/mL). AU was defined as an arbitrary unit identical to that of the sandwich enzyme immunoassay kit.

Compared to a CA215 sandwich enzyme assay, CA215 with a single RP215-specific epitope can be detected and no multiepitope is required to generate the required binding signal. However, the dynamic range of this CA215 competitive enzyme immunoassay is relatively narrow and restricted within a certain CA215 concentration range, when compared to that of the CA215 sandwich enzyme immunoassay for CA215 quantitation. Therefore, in this review, all the data related to CA215 levels are provided from the use of the CA215 sandwich enzyme immunoassay.

\section{Results and Discussion}

\section{Applications of CA215 and other known cancer biomarkers in routine cancer immunodiagnostics}

Early evaluation studies with ovarian and cervical cancer: Following the initial generation and characterization of RP215 in 1987, it was thought that the cognate antigen to RP215, CA215, is an antigen which was commonly found in ovarian and cervical tumors, as well as the sera of ovarian or cervical cancer patients. Therefore, the name of COX-1 was used during early studies of CA215 [4, 27]. However, by use of MALDI-TOF MS analysis, it was clearly demonstrated that CA215 consists mainly of immunoglobulin heavy chains expressed by cancer cells $[1,5$, 11, 18]. Nevertheless, serum CA215 levels among patients with cervical or ovarian cancer were investigated in great detail, particularly the stage-dependence of positive CA215 rates [1]. Typical results of such clinical immunodiagnostic studies are summarized in Table 1 for cervical and ovarian cancer. It was clearly demonstrated that positive rates correlated well with cancer diagnosis and clinical stage. Serum CA215 levels between cancer patients and normal individuals were found to be significantly different $(P<0.001)$. In the case of ovarian cancer, the positive rates range from $58-86 \%$ depending on clinical stage, when compared to normal individuals. For cervical cancer patients, the positive rates were as high as $66-94 \%$ at advanced cancer stages [1].

Through statistical analysis, the stage dependence of serum CA215 levels was clearly demonstrated for either ovarian or cervical cancer, especially at Stage I vs. Stage II or III $(P<0.001)$. 
Table 1: Serum levels of CA215 (in AU/mL) from Normal Individuals and Patients with Different Stages of Ovarian or Cervical Carcinoma.

\begin{tabular}{|c|c|c|c|c|c|c|c|}
\hline & \multirow{2}{*}{ Normal control } & \multicolumn{3}{|c|}{ Ovarian carcinoma } & \multicolumn{3}{|c|}{ Cervical carcinoma $^{\mathrm{b}}$} \\
\hline & & Stage 1 & Stage 2 & Stage 3 & Stage 1 & Stage 2 & Stage 3 \\
\hline Number of cases (n) & 59 & 24 & 7 & 40 & 25.0 & 20.0 & 5.0 \\
\hline Mean $(\mathrm{AU} / \mathrm{mL})^{\mathrm{c}}$ & 5.47 & 36.2 & 60.2 & 55.0 & 72.0 & 87.2 & 88.8 \\
\hline Standard deviation (SD) & 10.37 & 27.2 & 27.0 & 44.7 & 25.9 & 37.0 & 35.3 \\
\hline
\end{tabular}

a: for normal control vs. any of the stages 1,2 or 3 of ovarian carcinoma, $P<0.001$. For stage 1 vs. stage 2 and 3 ovarian carcinoma, $P<0.05$. For stage 2 vs. stage 3 ovarian carcinoma, $P>0.05$;

b: for normal control vs. any of the stages 1,2 , or 3 of cervical carcinoma, $P<0.001$. For stage 1 vs. stage 2 and 3 cervical carcinoma, $P<0.01$. For stage 2 vs. stage 3 cervical carcinoma, $P>0.05$.

c: AU represents an arbitrary unit which was defined based on the first generation enzyme immunoassay kit [1]. Modified from [1] with permission.

However, serum CA215 levels between Stage II and Stage III are not well differentiated $(P>0.05)$ [1]. Overall, these experimental results should serve to indicate that $\mathrm{CA} 215$ is indeed a reliable pan cancer biomarker for differential clinical diagnosis of patients with cervical or ovarian cancer at various clinical stages.

The results of these clinical studies with cervical and ovarian cancer also revealed that the mean serum CA215 levels remained at relatively high levels during the preoperative stages and within one week prior to surgical operations or chemo- or radiotherapy. In contrast, serum CA215 levels decreased significantly when determined seven days after surgical operations or chemo- or radiotherapy, as summarized in Figure 2 [1]. Based on these studies, it can be concluded that the surgical removal or chemo treatments of cancer among patients with cervical and ovarian cancer resulted in a statistically significant decrease in CA215 levels. The results indicate that CA215 detected from the cancer patients most likely originates from the tumor site and that the tumor burden can be adequately reflected by serum CA215 levels of cancer patients. Therefore, routine monitoring of cancer patients with serum CA215 levels should be beneficial regarding the status of cancer progression following scheduled therapeutic or surgical treatments.

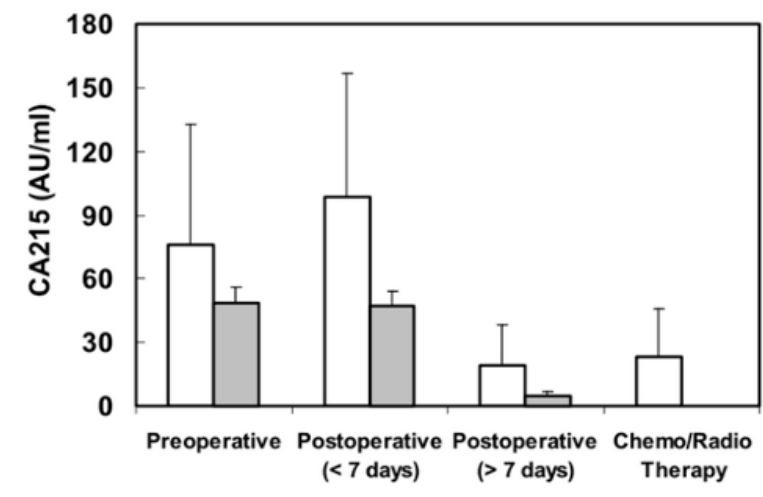

Figure 2: Comparisons of serum levels of CA215 among preoperative/ postoperative treatments as well as radio/chemo therapy for patients with ovarian or cervical carcinoma, displayed in histograms with standard deviations indicated by error bars. White: cervical carcinoma $(\mathrm{n}=$ 23), Grey: ovarian carcinoma $(\mathrm{n}=18)$, Preoperative vs. postoperative ( $>7$ days): $\mathrm{P}<0.05$, Preoperative vs. radio/chemo therapy ( $>7$ days): $\mathrm{P}$ $<0.05$. Taken from [1] with permission.
Based on the same RP215-based sandwich enzyme immunoassay, serum CA215 levels of more than 500 patients confirmed with either of twelve different cancers and were determined. Simultaneously, the clinical serum specimens were also evaluated for many other known -cancer biomarkers including Alpha-Fetoprotein (AFP),carcinoembryonic antigen (CEA), cancer antigen 125 (CA125), cancer antigen 15-3 (CA15-3), cancer antigen 19-9 (CA19-9), $\beta 2$-microglobulin, and cytokeratin 19 fragment (Cyfra 21-1) [21]. The positive rates for CA215 and other cancer biomarkers were determined and compared for each of the following types of cancer: lung, colon, liver, ovary, kidney, esophagus, pancreas, breast, and stomach. Results of this comparative study are summarized in Table 2.

Large scale evaluations of serum CA215 levels among cancer patients: In the case of serum CA215 levels, positive detection rates of $50 \%$ or higher were found in serum specimens of cancer patients with lymphoma or cancers of the lung, liver, esophagus, stomach, ovary, breast or cervix (Table 1) [21]. The scattergram of CA215 levels of different cancer types are presented in Figure 3 for comparisons. By using $0.1 \mathrm{AU} / \mathrm{mL}$ as the normal cut-off value, the positive rates of CA215 for different cancers were shown to be, respectively, $52 \%$ for lung cancer, $44 \%$ for colon cancer, $61 \%$ for esophageal cancer, $60 \%$ for stomach cancer, $59 \%$ for ovarian cancer, $71 \%$ for breast cancer, $38 \%$ for kidney cancer, $41 \%$ for pancreatic cancer, $51 \%$ for cervical cancer, and $83 \%$ for lymphoma [21]. Relative positive rates of serum CA215 with each cancer type are clearly demonstrated by the scattergram shown in Figure 3.

Clinical evaluations of cancer patient's specimens by using other known cancer biomarkers: Besides CA215, serum specimens were also used for quantitative determination of other known cancer biomarkers [21]. Among these cancer biomarkers, CA125 is most commonly used for the detection and monitoring of ovarian cancer [28, 29]. CA15-3 is most specific for breast cancer detection, and CA19-9 is more specific to cancer of the pancreas, stomach, and liver [30-33]. AFP is a known cancer biomarker which is specific to hepatoma and other germ cell tumors while CEA and $\beta 2$-microglobulin are more or less pan cancer biomarkers of broad tissue origins [34-36]. Cyfra21-1 was found to be unique to lung cancer, with positive detection rates of about $50 \%[37,38]$. In Table 2 , individual and combined positive rates are presented for six types of human cancer (lung, liver, ovary, esophagus, breast, and stomach) with the use of 
Table 2: Comparative Positive Detection Rates of Various Cancers by CA215-based and Other Cancer-Associated Antigen-based Enzyme Immunoassay Kits.

\begin{tabular}{|c|c|c|c|c|c|c|c|}
\hline $\begin{array}{l}\text { CA215 } \\
(0.1 \mathrm{AU} / \mathrm{mL})^{\mathrm{a}}\end{array}$ & & Lung (n) ${ }^{\text {b }}$ & Liver (n) & Ovary (n) & Esophagus (n) & Breast (n) & Stomach (n) \\
\hline \multirow{3}{*}{$\begin{array}{l}\text { CEA } \\
(5 \mathrm{ng} / \mathrm{ml})\end{array}$} & $I^{c}$ & $52 \%(112)$ & $74 \%(58)$ & \multirow{3}{*}{-} & $61 \%(23)$ & $71 \%(44)$ & $60 \%(30)$ \\
\hline & $\mathrm{II}^{\mathrm{d}}$ & $67 \%(33)$ & $54 \%(35)$ & & $47 \%(19)$ & $95 \%(20)$ & $50 \%(14)$ \\
\hline & III $^{\mathrm{e}}$ & $94 \%$ & $81 \%$ & & $65 \%$ & $96 \%$ & $70 \%$ \\
\hline \multirow{3}{*}{$\begin{array}{l}\text { AFP } \\
(20 \mathrm{ng} / \mathrm{ml})\end{array}$} & I & \multirow{3}{*}{-} & $74 \%$ (58) & \multirow{3}{*}{-} & \multirow{3}{*}{-} & \multirow{3}{*}{-} & \multirow{3}{*}{-} \\
\hline & II & & $50 \%(40)$ & & & & \\
\hline & III & & $85 \%$ & & & & \\
\hline \multirow{3}{*}{$\begin{array}{l}\text { CA125 } \\
(35 \mathrm{AU} / \mathrm{ml})\end{array}$} & I & $52 \%(112)$ & $74 \%(58)$ & $59 \%(68)$ & $61 \%(23)$ & \multirow{3}{*}{-} & \multirow{3}{*}{-} \\
\hline & II & $85 \%$ (13) & $85 \%$ (13) & $59 \%(66)$ & $50 \%(12)$ & & \\
\hline & III & $85 \%$ & $92 \%$ & $82 \%$ & $75 \%$ & & \\
\hline \multirow{2}{*}{$\begin{array}{l}\text { CA19-9 } \\
(37 \mathrm{AU} / \mathrm{ml})\end{array}$} & I & \multirow{2}{*}{-} & $74 \%(58)$ & \multirow{2}{*}{-} & \multirow{2}{*}{-} & \multirow{2}{*}{ - } & $60 \%(30)$ \\
\hline & III & & $82 \%$ & & & & $81 \%$ \\
\hline \multirow{3}{*}{$\begin{array}{l}\text { CA15-3 } \\
(30 \mathrm{AU} / \mathrm{ml})\end{array}$} & I & \multirow{3}{*}{-} & \multirow{3}{*}{-} & \multirow{3}{*}{ - } & \multirow{3}{*}{ - } & $71 \%(44)$ & \multirow{3}{*}{-} \\
\hline & II & & & & & $83 \%(6)$ & \\
\hline & III & & & & & $83 \%$ & \\
\hline \multirow{3}{*}{$\begin{array}{l}\beta_{2} \text { microglobulin }(2.6 \\
\mathrm{ng} / \mathrm{ml})\end{array}$} & I & \multirow{3}{*}{-} & $74 \%(58)$ & $59 \%(68)$ & \multirow{3}{*}{-} & \multirow{3}{*}{ - } & \multirow{3}{*}{ - } \\
\hline & II & & $56 \%(16)$ & $90 \%(10)$ & & & \\
\hline & III & & $81 \%$ & $100 \%$ & & & \\
\hline \multirow{3}{*}{$\begin{array}{l}\text { Cyfra21-1 } \\
(3.3 \mathrm{ng} / \mathrm{ml})\end{array}$} & I & $52 \%(112)$ & \multirow{3}{*}{-} & \multirow{3}{*}{ - } & \multirow{3}{*}{ - } & \multirow{3}{*}{ - } & \multirow{3}{*}{-} \\
\hline & II & $50 \%(52)$ & & & & & \\
\hline & III & $77 \%$ & & & & & \\
\hline \multicolumn{8}{|c|}{$\begin{array}{l}\text { a: the concentration in each bracket ( ) is the cut-off value for } \\
\text { b: n refers to the number of patient cases; } \\
\text { c: CA215 only; } \\
\text { d: other marker only; } \\
\text { e: III: combined, Modified taken from [21] with permission. }\end{array}$} \\
\hline
\end{tabular}

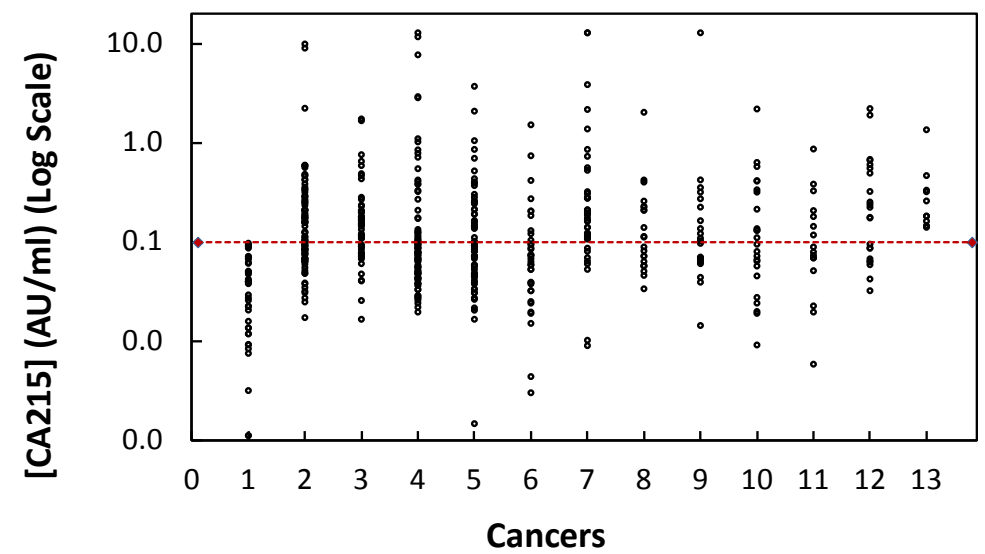

Figure 3: Scattergram to reveal the serum CA215 levels and positive rates from normal individuals $(\mathrm{n}=52)$ and cancer patients (total $\mathrm{n}=557) .1$ : normal individuals as the negative control $(5 \%, \mathrm{n}=52) ; 2$ : lung cancer $(52 \%, \mathrm{n}=112)$; 3 : liver cancer $(74 \%, \mathrm{n}=58) ; 4:$ colon cancer $(44 \%, \mathrm{n}=95) ; 5$ : ovarian cancer $(59 \%, \mathrm{n}=68)$; 6 : prostate cancer $(40 \%, \mathrm{n}=40)$; 7 : breast cancer $(71 \%, \mathrm{n}=44)$; 8: kidney cancer $(38 \%$, $\mathrm{n}=23)$; 9 : esophageal cancer $(61 \%, n=23) ; 10$ : stomach cancer $(60 \%, n=30) ; 11$ : pancreatic cancer $(41 \%, n=17) ; 12$ : cervical cancer $(51 \%, n=33) ;$ and $13:$ lymphoma $(83 \%, n$ =12). The dash line indicates the cut-off value of $0.1 \mathrm{AU} / \mathrm{mL}$. The positive rates of all different cancers were statistically significant with $\mathrm{P}<0.05$. The AU was defined based on the second generation enzyme immunoassay kit. Taken from [21] with permission. 
CA215 and in combination with either one of the other seven cancer biomarkers [21]. In view of somewhat restricted tissue specificity of some of the known cancer biomarkers, only parts of the data are selectively presented for comparative analysis with those of CA215.

Overall, the combined use of CA215 and either one of these cancer biomarkers were found to commonly result in a much higher detection rate to a given cancer. For example, in the case of lung cancer, a $52 \%$ positive detection rate in cancer patients was found with CA215 marker whereas with Cyfra21-1 marker, a $50 \%$ positive detection rate was obtained. When both markers were combined for diagnosis, the positive detection rate was found to be as high as 77\% [21]. Similarly for ovarian cancer, the combined positive rates for both CA215 and CA125 increased from $59 \%$ to $82 \%$ [21].

\section{General Conclusion}

Relative clinical utility of CA215 and other known biomarkers was assessed through large scale and extensive evaluations of serum specimens from cancer patients [21]. It has been wellestablished through early studies that CA215 consists mainly of immunoglobulins expressed by cancer cells both in vitro and in vivo $[1,5,11,18]$. Since RP215 was shown to recognize a carbohydrate-associated epitope in CA215, RP215 was utilized in the formulations of sandwich enzyme immunoassays for quantitative determination of serum CA215 levels of patients with all different types of cancer. As a pan cancer marker, CA215 is better than CEA or $\beta 2$-microglobulin and shows higher positive detection rates to most cancers [21]. CA215 is also comparable to CA125 in terms of positive detection rates for most human cancers, including ovarian cancer. However, in the case of cervical cancer, a much higher detection rate was found with the use of CA215 alone than that of CA125 (81\% vs. 13\%). However, combinations of CA215 and CA125 gave a better detection rate to ovarian cancer (59\% vs. 82\%) [21]. In the case of lung cancer, CA215 and Cyfra21-1 gave comparable positive detection rates [21]. Combinations of CA215 with either CEA or AFP appeared to give higher positive detection rates to liver cancer [21]. In summary, combinations of CA215 with other cancer biomarkers are certainly beneficial in increasing positive detection rates during routine clinical diagnosis of a given cancer. Therefore, the large scale evaluation studies of CA215 presented in this review have clearly demonstrated the potential clinical utility of CA215 as a pan cancer biomarker [21].

\section{Acknowledgements}

This research project was supported in parts by Sue fay Liu's Natural Sciences and Engineering Research Council of Canada (NSERC) undergraduate award and by the National Research Council of Canada Industrial Research Assistance Program (NRCIRAP) program (\#07119, \#090601, and \#703951). The excellent technical assistance of Sue fay Liu to this project is acknowledged.

\section{Declaration}

Gregory Lee is the co-founder of Vancouver Biotech Ltd. No conflict of interest is declared.

\section{References}

1. Lee G. Cancer cell-expressed immunoglobulins: CA215 as a pan cancer marker and its diagnostic applications. Cancer Biomark. 2009; 5(3): 137-142.

2. Lee G, Chu R-A, Ting HH. Preclinical assessment of anti-cancer drugs by using RP215 monoclonal antibody. Cancer Biol Ther. 2009; 8(2): 161-166.

3. Lee G, Wu Q, Li CH, Ting HH, Chien C-H. Recent studies of a new carbohydrate-associated pan cancer marker, CA215. Journal of Clinical Ligand Assay. 2006; 29(1): 47-51.

4. Lee CY, Chen KW, Sheu FS, Tsang A, Chao KC, Ng HT. Studies of a tumorassociated antigen, COX-1, recognized by a monoclonal antibody. Cancer Immunol Immunother. 1992; 35(1):19-26.

5. Lee G, Laflamme E, Chien C-H, Ting HH. Molecular identity of a pan cancer marker, CA215. Cancer Biology and Therapy. 2008; 7(12): 2007-2014.

6. Qiu X, Zhu X, Zhang L, Mao Y, Zhang J, Hao P, et al. Human epithelial cancers secrete immunoglobulin $G$ with unidentified specificity to promote growth and survival of tumor cells. Cancer Res. 2003; 63(19): 6488-6495.

7. Chen Z, Qiu X, Gu J. Immunoglobulin expression in non-lymphoid lineage and neoplastic cells. Am J Pathol. 2009; 174(4): 1139-1148.

8. Niu N, Zhang J, Guo Y, Zhao Y, Korteweg C, Gu J. Expression and distribution of immunoglobulin $\mathrm{G}$ and its receptors in the human nervous system. Int J Biochem Cell Biol. 2011; 43(4): 556-563.

9. Niu N, Zhang J, Wang S, Sun Y, Korteweg C, Gao W, et al. Expression and distribution of immunoglobulin $\mathrm{G}$ and its receptors in an immune privileged site: the eye. Cell Mol Life Sci. 2011; 68(14): 2481-2492.

10. Huang J, Zhang L, Ma T, Zhang P, Qiu X. Expression of immunoglobulin gene with classical V-(D)-J rearrangement in mouse testis and epididymis. J Histochem Cytochem. 2009; 57(4): 339-349.

11. Lee G, Zhu M, Ge B, Potzold S. Widespread expressions of immunoglobulin superfamily proteins in cancer cells. Cancer Immunol Immunother. 2012; 61(1): 89-99.

12. Lee G, Azadi P. Peptide mapping and glycoanalysis of cancer cellexpressed glycoproteins CA215 recognized by RP215 monoclonal antibody. J Carbohydr Chem. 2012; 31(1): 10-30.

13. Lee G, Ge B. Inhibition of in vitro tumor cell growth by RP215 monoclonal antibody and antibodies raised against its anti-idiotype antibodies. Cancer Immunol Immunother. 2010; 59(9): 1347-1356.

14. Lee G, Zhu M, Ge B, Cheung AP, Chien C-H, Chow S-N, et al. Carbohydrateassociated immunodominant epitope(s) of CA215. Immunol Invest. 2012; 41(3): 317-336.

15. Lee G, Cheung A, Ge B, Zhu M, Giolma B, Li B, et al. CA215 and GnRH receptor as targets for cancer therapy. Cancer Immunol Immunother. 2012; 61(10): 1805-1817.

16. Lee G, Zhu M, Ge B. Potential monoclonal antibody therapy for the treatment of ovarian cancer. In: Farghaly SA, editor. In: Ovarian Cancer - Basic Science Perspective. InTech. 2012; 385-406.

17. Lee G, Ge B. Implications of apoptosis in cancer immunotherapy. Adv Biosci Biotechnol. 2012; 3: 679-685.

18. Lee G, Ge B. Cancer cell expressions of immunoglobulin heavy chains with unique carbohydrate-associated biomarker. Cancer Biomark. 2009; 5(4):177-188. 
19. Ma C, Wang Y, Zhang G, Chen Z, Qiu Y, Li J, et al. Immunoglobulin G expression and its potential role in primary and metastatic breast cancers. Current molecular medicine. 2013; 13(3): 429-437.

20. Jiang C, Huang T, Wang Y, Huang G, Wan X, Gu J. Immunoglobulin G expression in lung cancer and its effects on metastasis. PLoS ONE. 2014; 9(5): e97359.

21. Lee G, Ge B, Huang TK, Zheng G, Duan J, Wang IH. Positive identification of CA215 pan cancer biomarker from serum specimens of cancer patients. Cancer Biomark. 2010; 6(2):111-117.

22. Lee G, Liu S. Roles of cancerous antigen receptors and CA215 in the innate immunity of cancer cells. Open Journal of Immunology. 2013; 3(3):127-138.

23. Lee G, Huang C-Y, Liu S, Zhang G. The immunology of cancer cells. SO Immunology. 2013; 1(1): 1-4.

24. Lee G, Huang C-Y, Tang Y, Zhang H. Potential roles of cancerous immunoglobulins in the immunology of cancer cells. J Clin Cell Immunol. 2014; 5(2):1-7.

25. Lee G, Huang C-Y, Liu S, Chien CH, Chow SN. Dual roles of cancer cellexpressed immunoglobulins in cancer immunology. Am J Immunol. 2014; 10(3):156-165.

26. Lee G. Cancerous immunoglobulins in cancer immunology. J Clin Cell Immunol. 2014; 5(6):1-8.

27. Lee G, Ng HT. Clinical evaluations of a new ovarian cancer marker COX-1. Int JGynaecol Obstet. 1995; 49: S27-32.

28. Kenemans P, Yedema CA, Bon GG, von Mensdorff-Pouilly S. CA 125 in gynecological pathology--a review. Eur J Obstet Gynecol Reprod Biol. 1993; 49(1-2):115-124.

29. Eagle K, Ledermann JA. Tumor markers in ovarian malignancies. Oncologist. 1997; 2(5): 324-329.
30. Duffy MJ, Shering S, Sherry F, McDermott E, O’Higgins N. CA 153: a prognostic marker in breast cancer. Int J Biol Markers. 2000; 15(4):330-333.

31. Goonetilleke KS, Siriwardena AK. Systematic review of carbohydrate antigen (CA 19-9) as a biochemical marker in the diagnosis of pancreatic cancer. Eur J Surg Oncol. 2007; 33(3): 266-270.

32. Ishigami S, Natsugoe S, Hokita S, Che X, Tokuda K, Nakajo A, et al Clinical importance of preoperative carcinoembryonic antigen and carbohydrate antigen 19-9 levels in gastric cancer. J Clin Gastroenterol. 2001; 32(1):41-44.

33. Maestranzi S, Przemioslo R, Mitchell H, Sherwood RA. The Effect of Benign and Malignant Liver Disease on the Tumour Markers CA19-9 and CEA. Ann Clin Biochem. 1998; 35(1): 99-103.

34. Johnson PJ. The role of serum alpha-fetoprotein estimation in the diagnosis and management of hepatocellular carcinoma. Clinics in Liver Disease. 2001; 5(1): 145-159.

35. Weinstein PS, Skinner M, Sipe JD, Lokich JJ, Zamcheck N, Cohen AS Acute-phase proteins or tumour markers: the role of SAA, SAP, CRP and CEA as indicators of metastasis in a broad spectrum of neoplastic diseases. Scand J Immunol. 1984; 19(3): 193-198.

36. Cooper EH, Plesner T. Beta-2-microglobulin review: its relevance in clinical oncology. Med Pediatr Oncol. 1980; 8(4): 323-334.

37. Rastel D, Ramaioli A, Cornillie F, Thirion B. CYFRA 21-1, a sensitive and specific new tumour marker for squamous cell lung cancer. Report of the first European multicentre evaluation. Eur J Cancer. 1994; 30(5): 601-606.

38. Stieber P, Hasholzner U, Bodenmüller H, Nagel D, Sunder-Plassmann L, Dienemann H, et al. CYFRA 21-1: a new marker in lung cancer. Cancer. 1993; 72(3): 707-713. 